\title{
Transport Properties of Dumbbell Molecules by Equilibrium Molecular Dynamics Simulations
}

\author{
Song Hi Lee \\ Deparment of Chemistrv, Kungsung Chiversin, Busan 608-736, Korea \\ Received December 15, 2003
}

\begin{abstract}
We presents new results for transport properties of dumbbell fluids by equilibrium molecular dỵnamics (EMD) simulations using Green-Kubo and Einstein formulas. It is evident that the interaction between dumbbell molecules is less attractive than that between spherical molecules which leads to higher diffusion and to lower friction. The calculated viscosity. however, is almost independent on the molecular elongation within statistical error bar. which is contradicted to the Stokes' law. The calculated thermal conductivity increases and then decreases as molecular elongation increases. These results of viscosity and thermal conductivity for dumbbell molecules by EMD simulations are inconsistent with the earlier results of those by non-equilibrium molecular dynamics (NEMD) simulations. The possible limitation of the Green-Kubo and Einstein formulas with regard to the calculations of viscosity and thermal conductivity for molecular fluids such as the missing rotational degree of freedom is pointed out
\end{abstract}

Key Words : Diffusion. Viscosity, Thermal conductivity, Dumbbell. Molecular dynamics simulation

\section{Introduction}

The earliest molecular dynanics (MD) sintulation method has been widely used to study the monatonic fluids. ${ }^{1-3}$ considering a Lemnard-Jones ( $\mathrm{LJ}$ ) potential as the interaction between pairs of particles, which has lead to a determination of several thermodynamic and transport properties. For argon. the calculated quantities agree within a few percent with the experimental data. The purpose of this comparison between computed and experimental values is not to demonstrate that the real potential is a LJ potential, but to establish that the proposed model has all the significant physical properties of a real system. Then the sumulated system can be considered as a reference for theoretical studies of equilibriun and transport properties.

The earliest MD calculations on polyatomic fluids have been carried out by Harp and Berne ${ }^{4}$ using a Stockmayertype potential to simulate $\mathrm{CO}$ and $\mathrm{N}_{2}$ and by Rahman and Stillinger ${ }^{5}$ to simulate $\mathrm{H}_{2} \mathrm{O}$. For diatonic (dumbbell) molecules. a very simple extension of the hard-sphere model is to consider a diatomic composed of two hard spheres fused together, but more realistic models involve continuous potentials. Thus. $\mathrm{N}_{2} . \mathrm{F}_{3} . \mathrm{Cl}_{2}$ etc. have been depicted as two 'Leinard-Jones atonts separated by a fixed bond length. ${ }^{7-9}$

Recently. Lee and Cunmings reported results of nonequilibriun molecular dynamics (NEMD) sinulations for shear viscosities of pure dumbbell fluids. spherical/dumbbell mixtures, and dumbbell/dumbbell mixtures. ${ }^{\text {la }}$ It was found that the interaction between dumbbell molecules is less attractive than that between spherical molecules which leads to lower viscosities as was observed in the experimental fact that the viscosity of normal alkanes is less than that of branched alkanes.

More recently. Tokumasu et $a$. have studied the effect of molecular elongation on the thermal conductivity of diatomic liquids using a NEMD method. ${ }^{\text {] }}$ It was found that the reduced themal conductivity increases as molecular elongation increases. Detailed analysis of the molecular contribution to the thermal conductivity revealed that the contribution of the flux caused by energy transport and by translational energy transfer to the themal conductivity is independent of the molecular elongation. and the contribution of the heat flux caused by rotational energy transfer to the thermal conductivity increases with the increase in the molecular elongation.

In the present paper. the dependence of transport properties of dumbbell molecules on molecular elongation was analyzed by equilibrium MD simulations. This paper is organized as follows: In Section II. we present the molecular models and MD simulation methods. We discuss our simulation results in Section III and present the concluding remarks in Section IV.

\section{Molecular Models and NpT MD Simulation Methods}

The dumbbell molecule is modeled as an atom-atom or site-site model of a diatomic molecule. The total interaction is a sum of pairwise contributions from distinct atoms $a$ in molecule $i$, at position $\mathbf{r}_{\mathrm{ia}}$, and $b$ in molecule $j$. at position $\mathbf{r}_{\mathrm{j} b}$

$$
u_{i j}\left(r_{i j}\right)=\sum_{a=1}^{2} \sum_{b=1}^{2} t_{a b}\left(r_{a b}\right)
$$

where $r_{a b}$ is the inter-site separation $r_{a b}=\left|\mathbf{r}_{j a}-\mathbf{r}_{j b}\right|$ and $t_{a b}$ is the pair potential acting between sites $a$ and $b$ :

$$
u_{a b}\left(r_{a b}\right)=4 \varepsilon_{l}\left[\left(\frac{\sigma_{d}}{r_{a b}}\right)^{12}-\left(\frac{\sigma_{a l}}{r_{a b}}\right)^{6}\right]
$$

Here $\sigma_{d}$ and $\varepsilon_{i l}$ are the Lennard-Jones (LJ) parameters for the dumbbell molecule. The interatomic separation in a dumbbell. $l$. is chosen such as the volume of the dumbbell 
molecule is the same as that of a sphere of diameter $\sigma_{s}$. Since the volume of a dumbbell of two spheres of dianteter $\sigma_{a\}}$ is given by

$$
V_{a}=\frac{1}{6} \pi \sigma_{a}^{3}+\frac{1}{4} \pi \sigma_{a l}^{2} l-\frac{1}{12} \pi l^{3}
$$

for a given interatomic separation $l=L \sigma_{d}, \sigma_{d}$ is determined by equating $V_{d}=V_{s}$, and can be expressed in the form of $\sigma_{d}=$ $c \sigma_{s}$. In this study. we have chosen as $L=0.1 / 12.1 / 6,1 / 4$. and $1 / 3$, and $c$ is determined as 1.0.0.9616.0.9289. 0.9010. and 0.8772 . respectively. The other LJ parameter $\varepsilon_{k\}}$ is chosen as $\varepsilon_{s} / 4$. The LJ parameters, $\sigma_{s}$ and $\varepsilon_{s}$, for spherical $A_{\Gamma}$ are chosen as $0.3405 \mathrm{~nm}$ and $0.996 \mathrm{l} \mathrm{kJ} / \mathrm{mol}$, respectively. All MD simulations in NVT and NpT ensembles were carried on 1728 molecules and fully equilibrated for at least 50,000 time steps of $10^{-15}$ second (1 fento second). The equilibrium properties were then averaged over 5 blocks of 20,000 time steps for a total of 100.000 time steps. and the configurations of molecules were stored every 10 time steps for analyses of structural and dynanic properties. The intermolecular potentials were subject to spherical cutoffs as follows: the cutoff distance was $2.5 \sigma_{s}$ for pure dumbbell fluids.

The equations of translational motion in NpT ensemble are given by

$$
\begin{aligned}
& \bar{r}_{i}=p_{i} / m_{i}+\dot{\varepsilon} r_{i} \\
& \bar{p}_{i}=F_{i}-\dot{\varepsilon} r_{i}-\alpha p_{i} \\
& \dot{V}=3 \dot{\varepsilon} V .
\end{aligned}
$$

The equations of rotational motion about the center of mass for molecular fluids in NpT ensemble are derived using quaternions ${ }^{12.13}$ follows:

$$
\begin{gathered}
T_{i}^{\prime}=A_{i} T_{i} \\
L_{i}^{p}=T_{i}^{p}-\alpha_{i} L_{i}^{p} \\
\omega_{i k}^{p}=\frac{L_{i k}^{p}}{I_{k}} . k=x, y, z
\end{gathered}
$$

$$
\left(\begin{array}{l}
\dot{q}_{i 1} \\
\dot{q}_{i 2} \\
\dot{q}_{i 3} \\
\dot{q}_{i+}
\end{array}\right)=\frac{1}{2}\left(\begin{array}{cccc}
-q_{i 3} & -q_{i 4} & q_{i 2} & q_{31} \\
q_{i+} & -q_{i 3} & -q_{i 1} & q_{i 2} \\
q_{i 1} & q_{i 2} & q_{i+} & q_{i 3} \\
-q_{i 2} & q_{i 1} & -q_{33} & q_{34}
\end{array}\right)\left(\begin{array}{c}
\omega_{i x}^{p} \\
w_{i 3}^{p} \\
0 \\
0
\end{array}\right) .
$$

$$
\begin{aligned}
& A_{i l 1}=-q_{i 1} q_{i 1}+q_{22} q_{i 2}-q_{33} q_{33}+q_{i 4} q_{i 4} \\
& A_{i 12}=2\left(q_{13} q_{34}-q_{i l} q_{i 2}\right) \\
& A_{i 13}=2\left(q_{i 2} q_{i 3}+q_{i l} q_{i 4}\right) \\
& A_{i=1}=-2\left(q_{l 1} q_{i}+q_{3} q_{i 4}\right) \\
& A_{i 22}=q_{i l} q_{i 1}-q_{i 2} q_{i 2}-q_{33} q_{i 3}+q_{i 4} q_{i 4} \\
& A_{i 33}=2\left(q_{i 2} q_{i 4}-q_{i 1} q_{33}\right) \\
& A_{331}=2\left(q_{s_{2}} q_{33}-q_{i 1} q_{24}\right) \\
& A_{332}=-2\left(q_{11} q_{33}+q_{2} q_{34}\right) \\
& A_{i 33}=-q_{11} q_{i 1}-q_{2} q_{i 2}+q_{33} q_{33}+q_{i 4} q_{34}
\end{aligned}
$$

where $L_{i}$ is the angular monentum of molecule $i, T_{i}$ is the torque on molecule $i$ in the laboratory frame, $L_{3}^{p}$ and $\omega_{l}^{p}$ are, respectively, the angular momentum and angular velocities of molecule $i$ in its principle axis frame. $\boldsymbol{A}_{\mathrm{j}}$ is the rotation matrix which transforms vectors from the laboratory frame to the principal axis frame of molecule $i . I_{k}$ is the principal axis of momenta of inertia of each molecule, and $q_{i k}, k=1,2.3,4$. are the quatemion parameters related to the Euler angles describing the orientation of molecule $i$ in the laboratory frame

$$
\begin{aligned}
& e_{i x}=2\left(q_{i 2} q_{i 3}-q_{i 1} q_{i 4}\right) \\
& e_{i y}=-2\left(q_{i 1} q_{i 3}+q_{i 2} q_{i 4}\right) \\
& e_{i 5}=-q_{i 1} q_{i 1}-q_{i 2} q_{i 2}+q_{i 3} q_{i 3}+q_{i 4} q_{i 4}
\end{aligned}
$$

where $e_{i \alpha}(\alpha=\mathrm{x} . \mathrm{y} . \mathrm{z})$ are the components of its orientational unit vector of molecule $i$. Note that the quatemions satisfy the nommalization $q_{i 1^{2}}+q_{i 2}{ }^{2}+q_{i 3}{ }^{2}+q_{i 4^{2}}{ }^{2}=1$. The use of quatemions leads to singularity free equations of motion. ${ }^{1213}$ The translational and rotational temperature constraint parameters $_{\mathrm{t}}$ and ${ }_{\mathrm{r}}$ are given by

and

$$
\alpha_{t}=\frac{\sum_{j}^{N} p_{i} \cdot F_{i}}{\sum_{i}^{N} p_{i}^{2}}-\dot{\varepsilon}
$$

$$
\alpha_{r}=\sum_{i}^{N}\left[L_{i} \cdot T_{i}\right] / \sum_{i}^{N} L_{i}^{2}
$$

The dilation rate is given by

$$
\dot{\varepsilon}=-\frac{\sum_{i \neq j}^{N}\left(r_{i j} \cdot p_{i j}\right)\left(\Phi_{i j}{ }^{\prime \prime}+\Phi_{i j}{ }^{\prime} / r_{i j}\right)}{m \sum_{i \neq j}^{N} r_{i j}^{2}\left(\Phi_{i j}{ }^{\prime \prime}+\Phi_{i j}{ }^{\prime} / r_{i j}\right)+9 p V},
$$

where $\Phi_{i j}$ is the interaction potentials between molecules $i$ and $j$. Putting $\dot{\varepsilon}=0$ recovers the NVT ensemble.

\section{Results and Discussion}

Thermodynamic properties for dumbbell molecules at $94.4 \mathrm{~K}$ in NVT ensemble ( $=1728$ and $\mathrm{V}=83.432 \mathrm{~mm}^{3}$ ) obtained from our EMD simulations are listed in Table 1 . The Lennard-Jones (LJ) energy and total energy decrease negatively with increasing interatomic separation in a dumbbell. This means that the interaction between dumbbell molecules becomes less attractive with the increase in the molecular elongation. The calculated pressure in NVT ensemble EMD sinulations, however, becomes negative and then increases positively as molecular elongation increases. Since the volume of the dumbbell molecule in various dumbbell systems is the same as that of a sphere of diameter $\sigma_{s}$ this pressure change might be due to the molecular shape of dumbbell molecules. ${ }^{10}$ Especially note that the modification from the sphere of diameter $\sigma_{\mathrm{s}}$ to the dumbbell system of $\mathrm{L}=1 / 12$. of which the interatomic distance is $0.0801 \sigma_{s}$, brings a huge pressure change from 40 atm to the negative pressure of -170 atm. In the cases of $L=1 / 4$ and $1 / 3$. the calculated pressures are positive due to the repulsive interaction 
Table 1. LJ energy ( $E_{i, l}$ in $\mathrm{kJ} / \mathrm{mol}$ ), total encrgy ( $E_{j, r}$ in $\left.\mathrm{kJ} / \mathrm{mol}\right)$. and pressure $(p$ in atm) of dumbbell molecules at $94.4 \mathrm{~K}$ in .VV T ensembles $\left(l^{\prime}=83.432 \mathrm{~nm}^{3}\right)$

\begin{tabular}{cccccc}
\hline Properties & $\mathrm{I}=0$ & $\mathrm{I}=\mathrm{l} / \mathrm{I} 2$ & $\mathrm{I}=\mathrm{l} / 6$ & $\mathrm{I}=\mathrm{I} / 4$ & $\mathrm{I}=1 / 3$ \\
\hline$E_{l,-}$ & $-5.383+0.084$ & $-4.793-0.016$ & $-4.166+0.015$ & $-3.658-0.014$ & $-3.218+0.013$ \\
$E_{t+1, t}$ & $-4.206+0.026$ & $-3.616-0.017$ & $-2.989+0.016$ & $-2.480-0.014$ & $-2.041+0.014$ \\
$p$ & $39.53+20.03$ & $-169.4-35.1$ & $-129.4+34.4$ & $79.68-33.96$ & $193.5+32.5$ \\
\hline
\end{tabular}

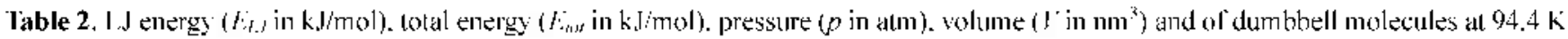
in Npl ensembles

\begin{tabular}{|c|c|c|c|c|c|}
\hline I'roperties & I. 0 & 1. $1 / 12$ & I. 166 & I. 14 & I. $1 / 3$ \\
\hline$b_{j S}$ & $-5.349 \pm 0.025$ & $-5.067=0.035$ & $-4.347 \pm 0.045$ & $-3.479=0.039$ & $-2.436 \pm 0.053$ \\
\hline$F_{\text {triti }}$ & $-4.172 \pm 0.026$ & $-3.890=0.035$ & $-3.169 \pm 0.046$ & $-2.302=0.039$ & $-1.258 \pm 0.053$ \\
\hline$p$ & $1.018 \pm 0.140$ & $1.024=0.494$ & $1.012=1.024$ & $1.027 \pm 1.177$ & $1.031 \pm 1.010$ \\
\hline$r^{-}$ & $84.21 \pm 0.35$ & $78.67=0.38$ & $79.93 \pm 0.61$ & $87.78 \pm 0.83$ & $111.2 \pm 3.5$ \\
\hline
\end{tabular}

between dumbbell molecules caused by the increase in the molecular elongation.

The corresponding NpT ensemble $(p=1$ atm) EMD simulations immediately carried out with a careful consideration of the long-range correction due to the spherical cut-off the potential, which is a tail correction estimating the contribution from pairs of particles whose distance apart is greater than the cut-off distance. ${ }^{1.15}$ Table 2 lists the resulting thermodynamic properties for dumbbell molecules at $94.4 \mathrm{~K}$ obtained from our ГMD simulations. The calculated pressures in NpT ensemble CMD simulations are much close to 1 atm. Again the modification from the sphere of diameter $\sigma_{s}$ to the dumbbell system of $l_{-}=1 / 12$ brings a huge volume change from $84 \mathrm{~nm}^{3}$ to $79 \mathrm{~nm}^{3}$. Also note that beyond the elongation of $[=1 / 3$, for example, in the dumbbell systems of $\mathrm{l}=5 / 12$ and $1 / 2$, the system becomes infinite dilution, keeping the pressure of the system as $1 \mathrm{~atm}$.

Figures 1 and 2 show the center of mass radial distribution functions of dumbbell molecules at $94.4 \mathrm{~K}$ in NVT and NpT

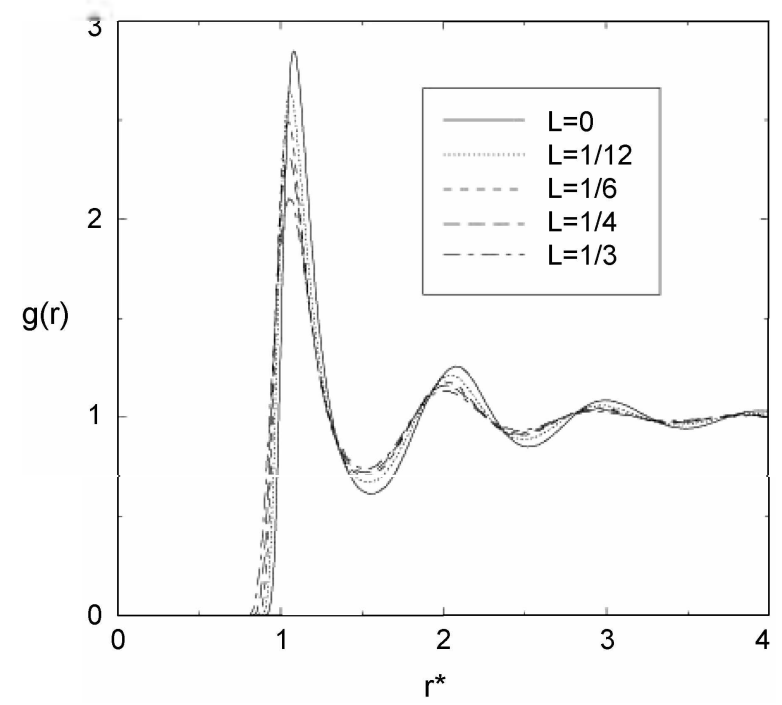

Figure 1. Center of mass radial distribution function of dumbbell molecules at $94.4 \mathrm{~K}$ in $\mathrm{NVT}$ ensembles $w$ ith $r^{*} r / \sigma_{*}$.

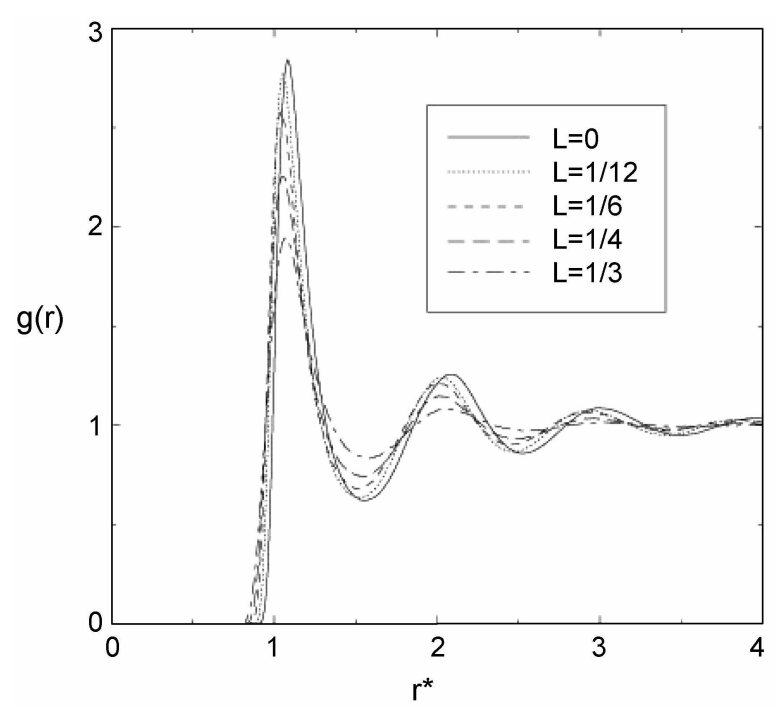

Figure 2. Center of mass radial distribution lunction of dumbbell molecules at $94.4 \mathrm{~K}$ in $\mathrm{Npl}$ ensembles with $r^{*}=r / \sigma$.

ensembles, respectively. The radial distribution function. $g(r)$, is defined as

$$
g(r)=\frac{1}{\rho_{0}}\left\langle\frac{N(r, \Delta r)}{V(r, \Delta \mu)}\right\rangle
$$

where $\rho_{0}$ is the bulk density, $N(r, \Delta r)$ is the number of molecules in a shell which is between $r-\Delta r / 2$ and $r+\Delta r / 2$ from the center of a molecule with $\Delta r=0.002 \mathrm{~nm}, V(r, \Delta r)$ is the volume of the shell, and $\langle\cdots\rangle$ indicates the corresponding ensemble (NVI or Np'l) average.

In both NVI and Npl ensemble EMD simulations, as the molecular elongation increases, the first and second peaks in the center of mass $g(r)$ diminish gradually and the minima increase. It is also observed that the nearest distance between centers of dumbbell molecules becomes shorter with the increase in the molecular elongation. [ This is because the centers of mass of dumbbell molecules come closer as the molecular elongation increases. When the center of mass $g(r)$ in NVI ensemble EMD is compared with that in Np' ensemble EMD for the same value of $L$, the difference in the 
first peaks of $g(\mu)$ indicates the volume change effect from NVT to NpT. At $\mathrm{L}=0$ the center of mass $g(r)$ is almost the same since volume change is so small. but in the cases of $\mathrm{L}=\mathrm{l} / 12$ and $1 / 6$, the decreasing volume makes the first peak of $g(r)$ higher, while in the cases of $L=1 / 3$ and $1 / 4$. the increasing volume makes the first peak of $g(\nu)$ lower.

As dynamic properties. we consider diffusion constant (D). viscosity (n), thermal conductivity $(\lambda)$, and friction constant ( $\zeta$ ) of dumbbell systems. Diffusion constant can be obtained through two routes: the Green-Kubo formula from velocity auto-correlation functions (VAC):

$$
D_{s}=\frac{1}{3} \int_{i j}^{\infty}\left\langle v_{i}(t) \cdot v_{i}(0)\right\rangle d t
$$

and the Einstein formula from mean square displacements (MSD):

$$
D_{s}=\frac{1}{6} \lim _{t \rightarrow \infty} \frac{d<|r(t)-r(0)|^{2}}{\mathrm{~d} t}
$$

Viscosity and thermal conductivity are calculated by the modified Green-Kubo and Einstein formulas for better statistical accuracy ${ }^{16 .}$

$$
\eta=\frac{V}{k T} \int_{0}^{\infty} d t \sum_{j}\left\langle P_{j \alpha \beta}(0) \cdot P_{j \alpha \beta}(t)\right\rangle
$$

where $P_{j \alpha \beta}(t)=\frac{1}{V}\left[m v_{i \alpha}(t) \cdot v_{j \beta}(t)+r_{j \alpha}(t) \cdot f_{i \beta}(t)\right]$ with $\alpha \beta=\mathrm{xy}, \mathrm{xz}, \mathrm{yx}, \mathrm{yz}, \mathrm{zx}, \mathrm{z}$, and the modified Einstein formula for shear viscosity is

$$
\eta=\frac{1}{2} \frac{V}{k T} \lim _{t \rightarrow \cdots} \frac{d}{d t} \sum_{j}\left\langle\left[Q_{i \alpha \beta}(t)-\underline{Q}_{i \alpha \beta}(0)\right]^{2}\right\rangle .
$$

where $Q_{i \alpha \beta j)}(t)-Q_{i \alpha \beta \beta}(0)=\int_{i}^{t} d \tau P_{j \alpha \beta \gamma}(\tau)$. The modified Green-Kubo formula for themal conductivity ${ }^{16}$ is

$$
\lambda=\frac{V}{k T^{2}} \int_{0}^{\infty} d t \sum_{j}\left\langle J_{Q, \alpha}(0)-J_{Q, \alpha}(t)\right\rangle
$$

where $J_{Q_{m}}(t)=\frac{1}{V}\left\{\varepsilon_{i}(t) \cdot v_{i c}(t)+r_{i \alpha}(t) \cdot\left[\mathbf{v}_{i}(t) \cdot f_{i}(t)\right]\right\}$ with $\alpha$ $=\mathrm{x}, y, \mathrm{z}$. and $\varepsilon_{i}(t)=\frac{p_{i}(t)^{2}}{2 m_{i}}+\frac{1}{2} \sum_{j \neq i} \phi\left[r_{i j}(t)\right]$. The modified Einstein formula for themal conductivity ${ }^{16}$ is

$$
\lambda=\frac{1}{2} \frac{V}{k T^{2}} \lim _{t \rightarrow \infty} \frac{d}{d t} \sum_{i}\left\langle\left[\varepsilon_{i \alpha}(t)-\varepsilon_{i \alpha}(0)\right]^{2}\right\rangle \text {. }
$$

where $\left(\varepsilon_{\gamma \alpha}(t)-\varepsilon_{\gamma \alpha}(0)\right)=\int_{i 1}^{t} d \tau J_{Q_{r \alpha}}(t)$. Friction constant is obtained from the time integral of the force auto-correlation function $^{17,18}$.

$$
\zeta=\frac{1}{\tau_{r}}=\frac{1}{3 k T} \int_{0}^{\tau} d t\left\langle f_{i}(t) \cdot f_{i}(0)\right\rangle .
$$

where $f_{i}(t)=F_{i}(t)-\left\langle F_{i}(t)\right\rangle . F_{i}(t)$ is the total force exerted on molecule $i$, and $\tau_{r}$ is the macroscopic relaxation time of the FAC. ${ }^{18}$ The friction constants were obtained by the time integral of the total FAC with choosing the upper limit of $\tau$ as the time which the FAC has the first negative value by assuming that the fast random force correlation ends at that time.

Diffusion constant. viscosity. and themal conductivity of dumbbell molecules through the Green-Kubo and Einstein formulas and friction constant at $94.4 \mathrm{~K}$ in NVT and NpT ensembles obtained from our EMD simulations are listed in Tables 3 and 4, respectively. In both NVT and NpT ensemble

Table 3. Diffusion constant ( $D$ in $\left.10^{-5} \mathrm{~cm}^{2} / \mathrm{sec}\right)$, viscosity $\left(\eta\right.$ in $\mathrm{mP}$ ), and themal conductivity ( $\lambda$ in $10^{-4} \mathrm{cal} / \mathrm{K} \cdot \mathrm{cm} \cdot \sec$ ) by the Green-Kubo and Einstein fornulas and friction constant ( $\zeta$ in $\mathrm{g} / \mathrm{mol} \mathrm{ps}$ ) of dumbbell molecules at $94.4 \mathrm{~K}$ in NVT ensembles

\begin{tabular}{ccccccc}
\hline \multicolumn{2}{c}{ Properties } & $\mathrm{L}=0$ & $\mathrm{~L}=1 / 12$ & $\mathrm{~L}=1 / 6$ & $\mathrm{~L}=1 / 4$ & $\mathrm{~L}=1 / 3$ \\
\hline $\mathrm{D}$ & $\mathrm{G}-\mathrm{K}$ & $2.433 \pm 0.086$ & $3.548 \pm 0.123$ & $4.225 \pm 0.127$ & $4.608 \pm 0.153$ & $4.917 \pm 0.108$ \\
& $\mathrm{E}$ & $2.429 \pm 0.062$ & $3.554 \pm 0.089$ & $4.254 \pm 0.104$ & $4.645 \pm 0.177$ & $4.922 \pm 0.118$ \\
$\eta$ & $\mathrm{G}-\mathrm{K}$ & $2.928 \pm 0.211$ & $2.908 \pm 0.284$ & $3.077 \pm 0.104$ & $3.015 \pm 0.120$ & $3.078 \pm 0.171$ \\
& $\mathrm{E}$ & $3.000 \pm 0.221$ & $2.896 \pm 0.170$ & $3.070 \pm 0.163$ & $2.987 \pm 0.236$ & $2.998 \pm 0.064$ \\
$\lambda$ & $\mathrm{G}-\mathrm{K}$ & $3.179 \pm 0.353$ & $3.202 \pm 0.243$ & $3.315 \pm 0.234$ & $3.189 \pm 0.211$ & $3.080 \pm 0.400$ \\
& $\mathrm{E}$ & $3.132 \pm 0.245$ & $3.175 \pm 0.219$ & $3.323 \pm 0.281$ & $3.119 \pm 0.207$ & $3.118 \pm 0.181$ \\
$\zeta$ & $\mathrm{Eq} .(12)$ & $182.6 \pm 0.8$ & $158.7 \pm 1.0$ & $144.6 \pm 1.0$ & $139.4 \pm 1.0$ & $136.2 \pm 0.4$ \\
& $\mathrm{Eq} .(13)$ & 322.6 & 221.2 & 185.8 & 170.3 & 159.6 \\
\hline
\end{tabular}

Table 4 . Diffusion constant $\left(D\right.$ in $\left.10^{-5} \mathrm{~cm}^{2} / \mathrm{sec}\right)$, viscosity $\left(\eta\right.$ in $\mathrm{mP}$ ), and themal conductivity $\left(\lambda\right.$ in $10^{-4} \mathrm{cal} / \mathrm{K} \cdot \mathrm{cm}$ sec) by the Green-Kubo and Einstein formulas and friction constant $(\zeta$ in $\mathrm{g} / \mathrm{mol} \cdot \mathrm{ps}$ ) of dumbbell molecules at $94.4 \mathrm{~K}$ in $\mathrm{NpT}$ ensembles

\begin{tabular}{ccccccc}
\hline \multicolumn{2}{c}{ Properties } & $\mathrm{L}=0$ & $\mathrm{~L}=1 / 12$ & $\mathrm{~L}=1 / 6$ & $\mathrm{~L}=\mathrm{l} / 4$ & $\mathrm{~L}=\mathrm{l} / 3$ \\
\hline $\mathrm{D}$ & $\mathrm{G}-\mathrm{K}$ & $2.590 \pm 0.098$ & $2.752 \pm 0.113$ & $3.646 \pm 0.059$ & $5.326 \pm 0.138$ & $9.187 \pm 0.073$ \\
& $\mathrm{E}$ & $2.516 \pm 0.093$ & $2.704 \pm 0.070$ & $3.645 \pm 0.083$ & $5.295 \pm 0.160$ & $9.302 \pm 0.224$ \\
7 & $\mathrm{G}-\mathrm{K}$ & $2.923 \pm 0.234$ & $3.031 \pm 0.364$ & $3.019 \pm 0.192$ & $2.967 \pm 0.283$ & $2.752 \pm 0.268$ \\
& $\mathrm{E}$ & $3.018 \pm 0.205$ & $3.190 \pm 0.318$ & $2.092 \pm 0.222$ & $2.874 \pm 0.206$ & $2.626 \pm 0.150$ \\
$\lambda$ & $\mathrm{G}-\mathrm{K}$ & $3.635 \pm 0.358$ & $3.855 \pm 0.487$ & $4.401 \pm 0.435$ & $4.393 \pm 0.507$ & $3.229 \pm 0.387$ \\
& $\mathrm{E}$ & $3.654 \pm 0.251$ & $3.845 \pm 0.312$ & $4.479 \pm 0.246$ & $4.301 \pm 0.349$ & $3.232 \pm 0.228$ \\
$\zeta$ & $\mathrm{Eq} \cdot(12)$ & $179.2 \pm 1.0$ & $176.2 \pm 1.1$ & $156.4 \pm 0.7$ & $128.4 \pm 0.6$ & $89.89 \pm 1.80$ \\
& $\mathrm{Eq} \cdot(13)$ & 303.0 & 285.2 & 215.3 & 147.4 & 85.43 \\
\hline
\end{tabular}


EMD simulations. it is evident that as the molecular elongation increases. the diffusion constant increases and the friction constant decreases. The friction constant $\zeta$ is related to the diffusion constant $\mathrm{D}$ by Stoke- Einstein relation:

$$
\zeta=k T / L \text {. }
$$

Table 2 contains the friction constants obtained from $\mathrm{Eq}$. (13) using D obtained from the Green-Kubo formula in Table 2. Both the friction constants give a correct qualitative trend: decrease with the increase in the molecular elongation. The calculated friction constants from the FAC's using Eq. (12) are always less than those obtained from Eq. (13) which reflects that the random FAC decays slower than the total FAC as described by Kubo (see Fig. 2 in Ref. 18), but the overall agreement is quite good in the same magnitude of order. When diffusion and friction constants in NVT ensenible EMD are compared with those in NpT ensenible EMD for the same value of $\mathrm{L}$. the differences in diffusion and friction constants also indicate the volume change effect from NVT to NpT. In the cases of $L=1 / 12$ and $1 / 6$. the decreasing volume makes diffusion constant lower and friction constant higher. while in the cases of $L=0,1 / 3$ and $1 / 4$. the increasing volume makes diffusion constant higher and friction constant lower.

On the other hand, the viscosity ( $\eta$ ) obtained from our NVT and NpT ensemble EMD simulations does not show any clear trend of dependency on the molecular elongation. According to the Stokes' relation for a spherical particle of radius $\sigma(\zeta=6 \pi \sigma \eta)$. $\eta$ should be proportional to $\zeta$. But the calculated viscosity in NVT ensemble EMD is almost independent on the molecular elongation within statistical error bar, while the calculated viscosity in $\mathrm{NpT}$ ensemble EMD decreases with the increase in the molecular elongation except $L=0$. This result is inconsistent with the NVT ensemble NEMD simulation result for dumbbell molecules at $\mathrm{T}=135 \mathrm{~K}$ and $\rho=1.034 \mathrm{~g} / \mathrm{cm}^{3}$, which reported the decrease of the viscosity with the increase in the molecular elongation for $L=0,1 / 5.1 / 3$. and $1 / 2$. $^{16}$ The main reason for this inconsistency may be due to the missing of the contribution of rotational degree of freedom to the viscosity of dumbbell molecules in EMD simulations through Green-Kubo and Einstein relations.

The thermal conductivity $(\lambda)$ obtained from our NVT and $\mathrm{NpT}$ ensemble EMD simulations increases and then decreases as molecular elongation increases. This result is also inconsistent with the NEMD simulation result for two-center LJ molecules, ${ }^{1]}$ which reported that the reduced thermal conductivity increases as molecular elongation increases. It was found that the contribution of the flux caused by energy transport and by translational energy transfer to the thernal conductivity is independent of the nolecular elongation. and the contribution of the heat flux caused by rotational energy transfer to the thermal conductivity increases with the increase in the molecular elongation. In the NEMD simulation. ${ }^{11}$ the rotational degree of freedom was taken into consideration in the heat flux of each liquid, but the heat flux, $J_{\operatorname{Oix}}(t)$ in $\mathrm{Eq}$. (1 la). is missing the term $\boldsymbol{\omega}(t) T_{i}(t)$, where $\boldsymbol{\omega}$ and $T_{j}$ are the angular velocity and the torque vector of molecule $i$, respectively. Also. the total energy $\boldsymbol{\varepsilon}_{(}(t)$ is missing the rotational kinetic energy term, $1 / 2 \boldsymbol{\omega} \cdot I_{i} \boldsymbol{\omega}$. where $I_{i}$ is the tensor of inertia of molecule $i$. This rotational degree of freedom might be taken into consideration in the viscous motion for dumbbell molecules. This should be pointed out as a possible limitation of the Green-Kubo and Einstein formulas with regard to the calculations of viscosity and thermal conductivity for molecular fluids. Recalculations of the viscosity and thermal conductivity, taking into consideration the rotational degree of freedom, for dumbbell molecules are presently under study.

\section{Conclusion}

The effect of molecular elongation on the transport properties of dumbbell fluids has been studied by NVT and NpT ensemble equilibrium molecular dynamics (EMD) simulations using Green-Kubo and Einstein fonmulas. The interaction between dumbbell molecules becomes less attractive with the increase in the molecular elongation. which leads to higher diffusion and to lower friction. The calculated diffusion and friction constants, $D$ and $\zeta$. are well described by StokeEinstein relation. The results of viscosity and thermal conductivity for dumbbell molecules obtained from our EMD simulations do not show any clear trend of dependency on the molecular elongation. But the earlier results using NEMD simulations reported that the viscosity decreases and the thermal conductivity increases as molecular elongation increases. It is pointed out that the early Green-Kubo and Einstein formulas for monatomic molecules is missing the rotational degree of freedom which should be taken into consideration for the transport properties of molecular fluids.

Acknowledgment. This research was supported by Kyungsung University Research Grants in 2004.

\section{References}

1. Alder. B. T.: Wainwright. T. E. J. Chem. Phus. 1959.31.459.

2. Rahmant. A. Plys Rav 1964. 1364. 405.

3. Berne, B. J.: Harp. G. D. Adwan. Chent. Phos 1970, 17.63.

4. Harp. G. D.: Beme. B. J. Phws. Rev 1970. 12,975

5. Rahman. A.; Stillinger. F. H. J. Chent. Phws. 1971, 55. 3336.

6. Streett. W. B.: Tildesley. D. J. Proc. R Soc. Lond 1976. \$348. 485.

7. Barojas. T.: Leresque. D.: Quentrec. B. Phus. Rev 1973. A7. 1092

8. Cheung. P. S. Y.: Powles. T. G. M Hol Plys. 1975. 30.921

9. Singer. K.: Taylor. A.: Singer. J. V. L. Mol Phys. 1977. 33, 1757.

10. Lee. S. H.: Cummings. P. T. M Iol. Sim. 2001. 27.139.

11. Tokumasu, T.; Ohara. T.: Kamijo, K. J. Chent. PIns. 2003, 118, 3677.

12. Evans. D. J. Hol Plys. 1977. 34.317.

13. Evans. D. J.: Murad. S. M fol. Phus. 1977. 34. 327.

14. Aller. M. P.: Tildesley. D. T. Computer Simulation of Liquids: Oxford Univ. Press: Oxford. 1987; p 64.

15. Lee. S. H.: Kim, H. S: Pak. H. J. Chent. Phus 1992, $97,6933$.

16. Lee. S. H; Park, D. K; Kang. D. B. Bull. Korean Chem. Soc. 2003. 24. 178.

17. Ciccotti. G.: Ferrario. M.: Hynes. T. T.: Kapral. R. J. Chem Plys. 1990. 93.7137.

18. Kubo. R. Rep. Prog Phws. 1966. 29,255 